\title{
Geochemical Characteristics and Quality of Groundwater Around Okemesi Fold Belt, South Western Nigeria
}

\author{
O. A. Okunlola ${ }^{1} \&$ A. A. Afolabi ${ }^{1}$ \\ ${ }^{1}$ Department of Geology University of Ibadan, Ibadan, Nigeria \\ Correspondence: O. A. Okunlola, Department of Geology University of Ibadan, Ibadan, Nigeria. E-mail: \\ o.okunlola@ui.edu.ng
}

Received: September 28, 2014

Accepted: October 9, 2014 Online Published: February 20, 2015

doi:10.5539/enrr.v5n1p46

URL: http://dx.doi.org/10.5539/enrr.v5n1p46

\begin{abstract}
This study involves the determination of the hydrochemical character and quality of springs, shallow and deep wells around the Okemesi fold belt, south western Nigeria. This is with a view to elucidate their nature, type and evaluate portability, and suitability for agricultural and industrial purposes. The Okemesi fold belt is underlain by schistose rocks, mainly mica schist, quartz schist, and quartzite with minor amphibolites and gneisses.

The major hydrochemical water type are $\mathrm{Na}-(\mathrm{K})-\mathrm{Cl}-\left(\mathrm{SO}_{4}\right)$ and $\mathrm{Ca}-(\mathrm{Mg})-(\mathrm{Cl})-\mathrm{SO}_{4}$ water facies constituting about $52 \%$ and $47 \%$ respectively. The study showed that almost all of the physico-chemical parameters such as total dissolved solid TDS $(18.75-790.50 \mathrm{mg} / \mathrm{l})$, electrical conductivity EC $(25-1054 \mu \mathrm{s} / \mathrm{cm})$, $\mathrm{pH}(5.4-7.4)$, temperature $\left(24.8-29.5^{\circ} \mathrm{C}\right)$, turbidity $(2.2-40.5$ N.T.U), total hardness $(10-274 \mathrm{mg} / \mathrm{l})$, total alkalinity (18 - $274 \mathrm{mg} / \mathrm{l})$, dissolved oxygen $(1.8-7 \mathrm{mg} / \mathrm{l})$, chlorine demand $(1.56-4.75 \mathrm{mg} / \mathrm{l})$; and bacteriological analysis (5 - $80 \mathrm{MPN}$ ) results were within the World Health Organization (WHO) limits recommended for drinking water. However, some groundwater samples have $\mathrm{Ni}^{+}$and $\mathrm{K}^{+}$concentrations slightly above the recommended standard.

These physico-chemical parameters, especially cations: $\mathrm{Ca}^{2+}(1.6-72.8 \mathrm{mg} / \mathrm{l}), \mathrm{Mg}^{2+}(0-4.39 \mathrm{mg} / \mathrm{l}), \mathrm{Na}^{+}(1.63-$ $75.0 \mathrm{mg} / \mathrm{l}), \mathrm{K}^{+}(0-108.3 \mathrm{mg} / \mathrm{l}), \mathrm{Si}(4.0-10 \mathrm{mg} / \mathrm{l}), \mathrm{Cu}(0-0.391 \mathrm{mg} / \mathrm{l}), \mathrm{Zn}(0-0.29 \mathrm{mg} / \mathrm{l}), \mathrm{Cd}(0-0 \mathrm{mg} / \mathrm{l}), \mathrm{Pb}(0-$ $0 \mathrm{mg} / \mathrm{l}), \mathrm{Fe}(0.1-0.1 \mathrm{mg} / \mathrm{l}), \mathrm{Ni}(0-0.043 \mathrm{mg} / \mathrm{l})$ and anions such as $\mathrm{HCO}_{3}(6.1-79.3 \mathrm{mg} / \mathrm{l}), \mathrm{Cl}^{-}(5-109 \mathrm{mg} / \mathrm{l}), \mathrm{SO}_{4}{ }^{2-}$ (38- $76 \mathrm{mg} / \mathrm{l}), \mathrm{NO}_{3}{ }^{2-}(0-0 \mathrm{mg} / \mathrm{l})$ and $\mathrm{PO}_{4}{ }^{3-}(0.005-0.03 \mathrm{mg} / \mathrm{l})$ seem to reflect the chemical nature of the underlying rock units suggesting dissolution, weathering and water - soil / rock interaction processes.
\end{abstract}

Keywords: springs, wells, physico-chemical, hydrochemical, quality

\section{Introduction}

As water percolates through the rocks, some ionic exchanges take place and invariably the water takes into solution in different concentrations some elemental composition of the rocks. (Abimbola et al., 2002) Therefore, the chemical property, and also bacteriological composition of groundwater is important amongst other criteria in the assessment of the quality of waters. While it is generally accepted that water bodies, rivers, lakes, dams and estuaries are continuously subject to dynamic state of change with respect to the geological age and geochemical characteristics (Adefemi \& Awokunmi, 2010), the availability of good quality water derived from these sources is an indispensable feature for preventing diseases and improving quality of life (Oluduro \& Adeyowe, 2007). It is now generally recognized that the quality of water is just as important as its quantity (Fan \& Steinberg, 1996; Gbodo \& Ogunyemi, 1999; Abimbola et al., 2002, 2008; Tredoux et al., 2001; Adelana \& Olasehinde, 2003; Adeyemi et al., 2003). Also apart from the fact that water quality is a function of physical, chemical and biological parameters, it is also subjective, as it depends on the particular intended use (Tijani, 1994). Therefore, water quality standards differ for various uses such as domestic (drinking), agriculture (irrigation) and industries.

The most significant geo hydraulic influence on groundwater chemistry arises from the source and circulation of groundwater itself (Amadi et al., 1989). Natural water contains some types of impurities whose nature and amount vary with source of water (Adeyemi et al., 2003). Metals for example, are introduced into aquatic system through several ways which include, weathering of rocks and leaching of soils, dissolution of aerosol particles from the atmosphere and from several human activities, including mining, processing and the use of metal based materials (Ipinmoroti, 1993; Adeyeye, 1994; Asaolu et al., 1997). 
There has been some studies on rock composition, structure and association around Okemesi, southwestern Nigeria (Okunlola \& Okoroafor, 2009; Ayodele \& Odeyemi, 2010;) but little or none has been carried out on the quality of water in this region Hence the need for this study, which is aimed at elucidating the nature, origin and uses of the springs, shallow and deep well water in the study area..This involves the determination of the the physical and chemical properties together with bacteriological load in the groundwater.

The petrogenetic features of the schistose rocks around the Okemesi fold belt area of investigation, which is part of the Ife-Ilesha schist belt has been described as one of the most complex lithological and structural frameworks amongst the Nigeria's metasedimentary belts (Olobaniyi, 2003; Okunlola \& Okoroafor, 2009).

Okemesi is located in areas of high relief, (Figure 1) as a result, runoff is high and infiltration rates are low. Hence, some of the hand dug wells and boreholes are concentrated at the center of the fold belt, while the rivers and the springs follow a dendritic pattern (Figure 1).
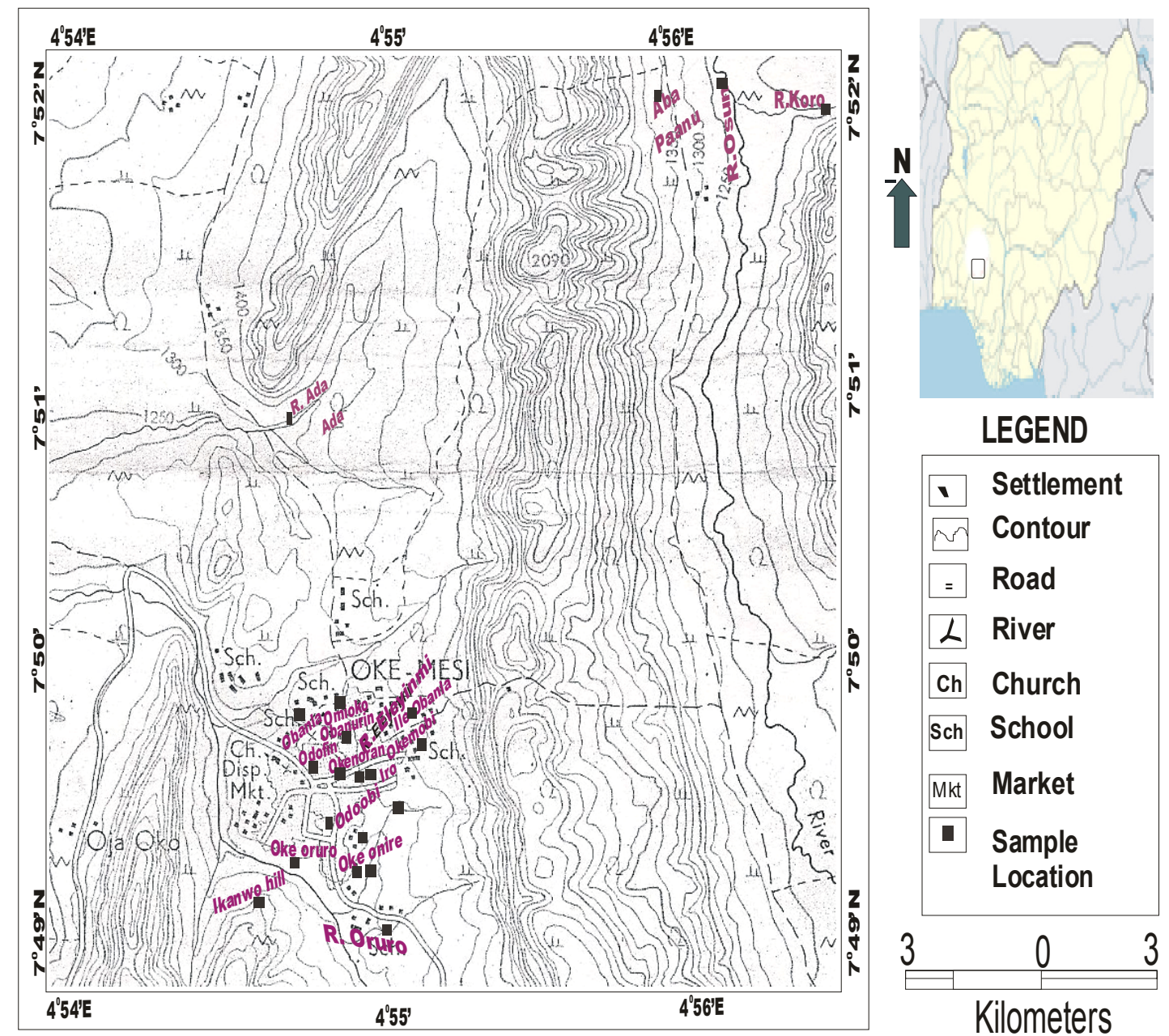

Figure 1. Map of sampling locations of surface and sub surface waters around Okemesi

The area of investigation lies completely within the 1: 50,000 sheet 253 (Ilesha NE) between latitude $7^{0} 49^{\prime}-7^{0} 52^{\prime}$ and longitude $4^{\circ} 54^{\prime}-4^{\circ} 56^{\prime}$, with the elevation ranging from 318 - 484m above the sea level. Okemesi is the largest town in the area. Other settlements in this area include Ajindo, Koro and Ada. The area is a planned settlement in spite of its rugged relief (Figure 1) and well serviced by a network of roads and footpaths (Figure 2).

A dendritic drainage system, upon which a trellis pattern has been super imposed mainly due to structural configuration characterize the area. Few large rivers and consequent streams that have their source from the nearby springs, together with deep and shallow wells present, are perennial, while small streams are seasonal with little or no meandering system (Figure 3). They flow westerly and a few northerly. The springs flow in a radial manner 
away from central range of schistose outcrops of Okemesi fold belt. Specifically, rivers like Oruro, Eleyinmi and Koro have utilized fractures in the area and cut across the divide.

Geomorphologically, the area forms an oval topographic feature with enclosing schistose and the surrounding gneissic complex (Figure 4). The range is broken in places by rivers, like Osun and Ada which have cut steep valleys and run almost at right angles to the trend of the range. The climate is typically equatorial, hot, dry and wet with mean monthly temperature around $27^{\circ} \mathrm{C}\left(80^{\circ} \mathrm{F}\right)$. Cloudiness and heavy precipitation help to moderate the daily temperature. It experiences about seven months (April-November) of torrential rain and associated high runoff and erosion, while December to March are often characterized by dry conditions.

\subsection{Geological Setting:}

Geologically, the study area is around the Okemesi fold belt of Ife - Ilesha schist belt within the Pre - Cambrian to Late Proterozoic Basement Complex rocks of southwestern Nigeria which in turn is part of the Pan - African mobile belt lying east of West African Craton (Jones \& Hockey, 1964; Odeyemi, 1976; Rahaman, 1976). The major rock units in the area of investigation are ampibolite, banded gneiss, mica schist, quartz schist and quartzite (Okunlola \& Okoroafor, 2009) (Figure 4). The details of the geological setting are contained in the aforementioned reference.

The basement rocks are commonly considered as poor aquifers because of their crystalline nature which leads to low porosity and permeability. However, appreciable porosity and permeability are developed through fracturing and weathering of the rocks (Davis \& Deweist, 1966). This makes an otherwise barren rock to function as groundwater aquifer (Abimbola et al., 2002). The rocks in the area of investigation have undergone different tectonic episodes and have as a result responded differently to tectonic deformation that has affected the terrain, hence the numbers and sizes of lineaments (fractures) (Ayodele \& Odeyemi, 2010) giving rise to the springs.

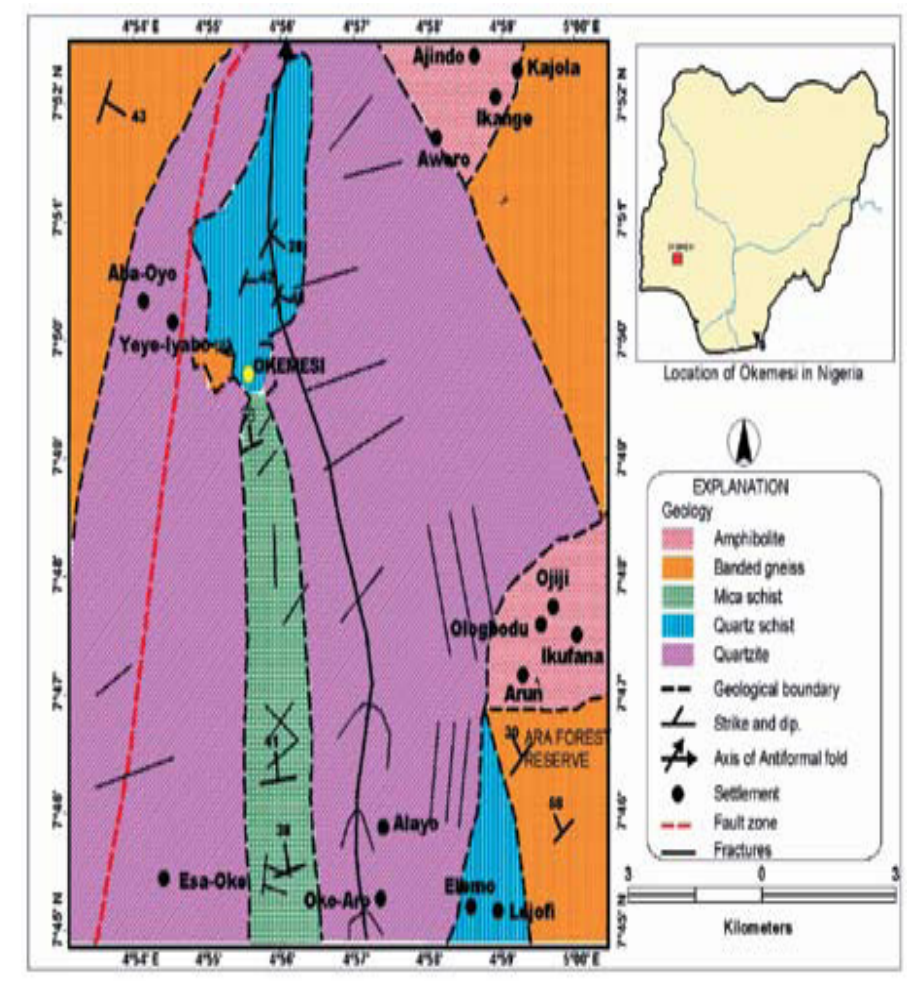

Figure 2. Geological map of Okemesi fold belt (after Okunlola \& Okoroafor, 2009)

\section{Materials and Methods}

A total of twenty one representative samples of surface and groundwater were collected around Okemesi, Koro, Ajindo and Ada. Comprising 2 springs, 10 hand dug wells, 5 boreholes and 4 rivers which constitute the major water supply for the inhabitant. Sampling was done before the onset of heavy rain to minimize dilution effect. At each location, an aliquot of water to be sampled was used to rinse the plastic container to avoid contamination before collecting the water into 2 well drained separate clean new set of plastic containers or bottles, one for the 
determination of anions while the other set of samples were for cations and these were acidified immediately with two drops of $\mathrm{HNO}_{3}$ in order to prevent loss of metals, bacterial and fungal growth. The first set was also stored in the refrigerator before bacteriological analysis.

The electrical conductivity, $\mathrm{pH}$ and temperature as a sensitive physico-chemical parameters were determined directly in the field using digital Hack $\mathrm{pH}$ meter while the location coordinates and elevation were determined by Ground Positioning System (GPS). Turbidity was determined by hack turbidity meter. Total hardness, total alkalinity, chlorine demand, dissolved oxygen, $\mathrm{NO}_{3}{ }^{2-}$ and $\mathrm{SO}_{4}{ }^{2-} \mathrm{Ca}^{2+}, \mathrm{Mg}^{2+}, \mathrm{Fe}^{3+}, \mathrm{HCO}_{3}$ and silica were determined by titrimetry method at the Central Laboratory, Water Corporation of Oyo State, Nigeria. The laboratory method used for the detection and enumeration of total coliform count is the multiple tube method.

Cations such as $\mathrm{K}^{+}$and $\mathrm{Na}^{+}$together with the trace elements such as $\mathrm{Cu}, \mathrm{Zn}, \mathrm{Cd}, \mathrm{Pb}$, and $\mathrm{Ni}$ were analyzed using the Atomic Absorption Spectrometer (Bulk scientific 210/211 VGP AAS), 220GF Graphite Furnace and 220AS Autosampler at the Agronomy laboratory, University of Ibadan, Oyo state, Nigeria while $\mathrm{PO}_{3}{ }^{2-}$ was analyzed by Ascorbic Acid method before determining its content in the solution by Spectrophotometer 70 at $882 \mathrm{~m} \mu$. The detection limits of the metals are $0.001 \mathrm{mg} / 1$ while for $\mathrm{K}^{+}$and $\mathrm{Na}^{+}$is $0.01 \mathrm{mg} / 1$

\section{Results and Discussion}

The data for physical, chemical and bacteriological parameters of both groundwater and surface water in the study area are presented in the Tables 1,2,3 and 4 respectively.

Table 1. The result of physical characteristics of surface water and groundwater around Okemesi

\begin{tabular}{|c|c|c|c|c|c|c|c|c|c|c|c|c|}
\hline $\begin{array}{l}\text { SAMPLE } \\
\text { ID }\end{array}$ & NORTHINGS & EASTINGS & $\begin{array}{l}E \\
(m)\end{array}$ & $\begin{array}{l}\text { TDS } \\
(\mathrm{mg} / \mathrm{l})\end{array}$ & $\begin{array}{l}\text { EC } \\
\mu \mathrm{S} / \mathrm{cm}\end{array}$ & pH & $\begin{array}{l}\mathrm{T} \\
\left({ }^{\circ} \mathrm{C}\right)\end{array}$ & $\begin{array}{l}\text { TU } \\
\text { (N.T.U.) }\end{array}$ & $\begin{array}{l}\text { TH } \\
(\mathrm{mg} / \mathrm{l})\end{array}$ & $\begin{array}{l}\text { TA } \\
(\mathrm{mg} / \mathrm{l})\end{array}$ & $\begin{array}{l}\text { DO } \\
(\mathrm{mg} / \mathrm{l})\end{array}$ & $\begin{array}{l}\mathrm{CD} \\
(\mathrm{mg} / \mathrm{l})\end{array}$ \\
\hline RL1 & $\mathrm{N} 7^{\circ} 50^{\prime} 59^{\prime \prime}$ & $\mathrm{E} 4^{\circ} 55^{\prime} 6.8^{\prime \prime}$ & 318 & 42.0 & 56 & 7.4 & 25.5 & 15.2 & 20 & 30 & 6.7 & 2.296 \\
\hline WL2 & $N 7^{\circ} 49^{\prime} 27^{\prime \prime}$ & $\mathrm{E} 4^{\circ} 55^{\prime} 31.6^{\prime \prime}$ & 402 & 114.75 & 153 & 6.5 & 29.5 & 6.8 & 68 & 68 & 4.4 & 2.169 \\
\hline RL3 & $N 7^{\circ} 52^{\prime} 46^{\prime \prime}$ & E45' $37.7^{\prime \prime}$ & 380 & 19.35 & 129 & 7.4 & 25.8 & 7.5 & 32 & 66 & 3.8 & 4.75 \\
\hline WL4 & $N 7^{\circ} 52^{\prime} 49^{\prime \prime}$ & $\mathrm{E} 4^{\circ} 56^{\prime} 32.7^{\prime \prime}$ & 398 & 51.0 & 68 & 6.0 & 26.3 & 14.2 & 50 & 38 & 4.3 & 1.585 \\
\hline RL5 & $\mathrm{N} 7^{\circ} 52^{\prime} 19^{\prime \prime}$ & $\mathrm{E} 4^{\circ} 58^{\prime} 11.5^{\prime \prime}$ & 441 & 401.25 & 535 & 6.7 & 25.4 & 14.2 & 252 & 274 & 1.8 & 2.196 \\
\hline WL6 & $N 7^{\circ} 49^{\prime} 27^{\prime \prime}$ & $\mathrm{E} 4^{\circ} 55^{\prime} 14.9^{\prime \prime}$ & 395 & 179.25 & 239 & 6.5 & 27.6 & 5 & 40 & 18 & 3.2 & 2.308 \\
\hline RL8 & $\mathrm{N} 7^{\circ} 49^{\prime} 22^{\prime \prime}$ & $\mathrm{E} 4^{\circ} 55^{\prime} 22^{\prime \prime}$ & 396 & 50.25 & 67 & 6.5 & 25.0 & 2.2 & 30 & 24 & 4.0 & 2.558 \\
\hline SPL9 & $\mathrm{N} 7^{\circ} 49^{\prime} 15^{\prime \prime}$ & $\mathrm{E} 4^{\circ} 55^{\prime} 3.8^{\prime \prime}$ & 484 & 18.75 & 25 & 5.4 & 24.8 & 28.2 & 16 & 18 & 5.4 & 2.67 \\
\hline WL10 & $\mathrm{N} 7^{\circ} 49^{\prime} 20^{\prime \prime}$ & $\mathrm{E} 4^{\circ} 55^{\prime} 21.8^{\prime \prime}$ & 396 & 113.25 & 151 & 5.6 & 25.5 & 32.5 & 54 & 36 & 3.6 & 2.919 \\
\hline BL11 & N749' 21" & E4 $55^{\circ} 13.6^{\prime \prime}$ & 414 & 53.25 & 71 & 6.2 & 27.0 & 2.6 & 26 & 36 & 6.4 & 2.121 \\
\hline BL12 & $N 7^{\circ} 49^{\prime} 12^{\prime \prime}$ & E4 $55^{\prime} 19.7^{\prime \prime}$ & 421 & 37.5 & 50 & 6.1 & 28.0 & 2.2 & 10 & 28 & 7.0 & 1.946 \\
\hline WL13 & $\mathrm{N} 7^{\circ} 50^{\prime} 04^{\prime \prime}$ & $\mathrm{E} 4^{\circ} 55^{\prime} 27.3^{\prime \prime}$ & 420 & 150.75 & 201 & 6.3 & 27.2 & 2.5 & 52 & 22 & 6.4 & 1.696 \\
\hline SPL14 & $\mathrm{N} 7^{\circ} 50^{\prime} 08^{\prime \prime}$ & $\mathrm{E} 4^{\circ} 55^{\prime} 28.3^{\prime \prime}$ & 443 & 43.4 & 62 & 6.0 & 25.3 & 8.4 & 16 & 32 & 3.7 & 1.946 \\
\hline WL15 & $N 7^{\circ} 49^{\prime} 53^{\prime \prime}$ & $\mathrm{E} 4^{\circ} 55^{\prime} 37.7^{\prime \prime}$ & 431 & 123.75 & 165 & 5.7 & 27.5 & 2.2 & 38 & 20 & 5.5 & 2.162 \\
\hline RL16 & $\mathrm{N} 7^{\circ} 49^{\prime} 45^{\prime \prime}$ & $\mathrm{E} 4^{\circ} 55^{\prime} 33.0^{\prime \prime}$ & 399 & 104.25 & 139 & 6.1 & 25.8 & 17.6 & 46 & 138 & 6.9 & 2.296 \\
\hline BL17 & $N 7^{\circ} 49^{\prime} 46^{\prime \prime}$ & $\mathrm{E} 4^{\circ} 55^{\prime} 27.3^{\prime \prime}$ & 408 & 642 & 856 & 6.0 & 26.8 & 7.5 & 274 & 144 & 3.4 & 1.56 \\
\hline BL19 & $N 7^{\circ} 49^{\prime} 55^{\prime \prime}$ & E4 $55^{\prime} 19.4^{\prime \prime}$ & 414 & 790.5 & 1054 & 6.0 & 27.5 & 4.6 & 158 & 60 & 5.0 & 2.196 \\
\hline WL20 & $N 7^{\circ} 49^{\prime} 54^{\prime \prime}$ & $\mathrm{E} 4^{\circ} 55^{\prime} 20.3^{\prime \prime}$ & 413 & 511.5 & 682 & 6.3 & 27.9 & 2.2 & 224 & 184 & 2.4 & 2.169 \\
\hline WL21 & $\mathrm{N} 7^{\circ} 50^{\prime} 06^{\prime \prime}$ & $\mathrm{E} 4^{\circ} 55^{\prime} 27.9^{\prime \prime}$ & 413 & 75 & 100 & 6.5 & 25.7 & 40.5 & 30 & 54 & 3.7 & 2.308 \\
\hline WL23 & $\mathrm{N} 7^{\circ} 49^{\prime} 57^{\prime \prime}$ & $\mathrm{E} 4^{\circ} 55^{\prime} 23.3^{\prime \prime}$ & 414 & 472.5 & 630 & 6.4 & 27.8 & 2.3 & 90 & 84 & 3.4 & 2.296 \\
\hline WL25 & $N 7^{\circ} 49^{\prime} 44^{\prime \prime}$ & $\mathrm{E} 4^{\circ} 55^{\prime} 25.2^{\prime \prime}$ & 417 & 402.75 & 537 & 7.2 & 28.7 & 30.4 & \multicolumn{2}{|l|}{164} & 5.0 & 2.196 \\
\hline \multicolumn{2}{|c|}{ RL- river location } & \multicolumn{3}{|c|}{ E- elevation } & & \multicolumn{3}{|c|}{ TU- turbidity } & \multicolumn{4}{|c|}{ TH- total hardness } \\
\hline WL- wel & location & \multicolumn{4}{|c|}{ TDS- total dissolve solid } & $\mathrm{T}-\mathrm{t}$ & nper & ture & \multicolumn{4}{|c|}{ DO- dissolved oxygen } \\
\hline BL- bore & ole location & \multicolumn{4}{|c|}{ EC- electrical conductivity } & $\mathrm{CD}$ & chlor & ne demar & \multicolumn{4}{|c|}{ SPL- spring location } \\
\hline SAR- soc & ium absorptios & ratio $\mathrm{TA}-\mathrm{tc}$ & tal al & linity & & $\mathrm{S} / \mathrm{N}$ & erial & number & & & & \\
\hline
\end{tabular}


Table 2. The result of chemical characterstics (cations and trace metals) of surface water and groundwater around okemesi

\begin{tabular}{|c|c|c|c|c|c|c|c|c|c|c|}
\hline SAMPLE ID & $\begin{array}{l}\mathrm{Ca}^{2+} \\
(\mathrm{mg} / \mathrm{l})\end{array}$ & $\begin{array}{l}\mathrm{Mg}^{2+} \\
(\mathrm{mg} / \mathrm{l})\end{array}$ & $\begin{array}{l}\mathrm{Na}^{+} \\
(\mathrm{mg} / \mathrm{l})\end{array}$ & $\begin{array}{l}\mathrm{K}^{+} \\
(\mathrm{mg} / \mathrm{l})\end{array}$ & $\begin{array}{l}\mathrm{Cu} \\
(\mathrm{mg} / \mathrm{l})\end{array}$ & $\begin{array}{l}\mathrm{Zn} \\
(\mathrm{mg} / \mathrm{l})\end{array}$ & $\begin{array}{l}\text { Cd } \\
(\mathrm{mg} / \mathrm{l})\end{array}$ & $\begin{array}{l}\text { Pb } \\
(\mathrm{mg} / \mathrm{l})\end{array}$ & $\begin{array}{l}\mathrm{Fe} \\
(\mathrm{mg} / \mathrm{l})\end{array}$ & $\begin{array}{l}\begin{array}{l}\mathrm{Ni} \\
(\mathrm{mg} / \mathrm{l})\end{array} \\
\end{array}$ \\
\hline RL1 & 4.80 & 0.195 & 5.23 & 2.11 & 0.039 & 0.000 & ND & ND & $<0.1$ & 0.015 \\
\hline WL2 & 16.0 & 0.683 & 6.86 & 8.49 & 0.021 & 0.000 & ND & ND & $<0.1$ & 0.014 \\
\hline RL3 & 9.60 & 0.195 & 8.56 & 6.17 & 0.016 & 0.006 & ND & ND & $<0.1$ & 0.004 \\
\hline WL4 & 12.0 & 0.488 & 6.95 & $8 . .27$ & 0.391 & 0.080 & ND & ND & $<0.1$ & 0.043 \\
\hline RL5 & 28.8 & 4.390 & 25.3 & 1.43 & 0.018 & 0.000 & ND & ND & $<0.1$ & 0.006 \\
\hline WL6 & 9.60 & 0.390 & 22.00 & 16.20 & 0.016 & 0.006 & ND & ND & $<0.1$ & 0.004 \\
\hline RL8 & 4.00 & 0.488 & 6.120 & 3.68 & 0.020 & 0.000 & ND & ND & $<0.1$ & 0.025 \\
\hline SPL9 & 4.00 & 0.146 & 1.630 & 0.000 & 0.036 & 0.001 & ND & ND & $<0.1$ & 0.007 \\
\hline WL10 & 21.6 & 0.000 & 4.100 & 3.860 & 0.037 & 0.027 & ND & ND & $<0.1$ & 0.011 \\
\hline BL11 & 4.80 & 0.342 & 3.550 & 1.870 & 0.000 & 0.005 & ND & ND & $<0.1$ & 0.014 \\
\hline BL12 & 1.60 & 0.146 & 2.760 & 4.660 & 0.002 & 0.005 & ND & ND & $<0.1$ & 0.000 \\
\hline WL13 & 12.0 & 0.537 & 4.450 & 25.40 & 0.017 & 0.000 & ND & ND & $<0.1$ & 0.018 \\
\hline SPL14 & 1.60 & 0.293 & 6.300 & 1.400 & 0.066 & 0.001 & ND & ND & $<0.1$ & 0.000 \\
\hline WL15 & 28.8 & 0.439 & 17.70 & 4.92 & 0.032 & 0.013 & ND & ND & $<0.1$ & 0.033 \\
\hline RL16 & 8.00 & 0.439 & 8.840 & $2 . .960$ & 0.014 & 0.001 & ND & ND & $<0.1$ & 0.018 \\
\hline BL17 & 72.8 & 2.245 & 34.00 & 23.60 & 0.019 & 0.020 & ND & ND & $<0.1$ & 0.028 \\
\hline BL19 & 36.0 & 1.650 & 75.00 & 108.3 & 0.004 & 0.028 & ND & ND & $<0.1$ & 0.029 \\
\hline WL20 & 64.0 & 1.562 & 32.10 & 48.20 & 0.048 & 0.000 & ND & ND & $<0.1$ & 0.033 \\
\hline WL21 & 5.60 & 0.390 & 7.050 & $3 . .320$ & 0.051 & 0.007 & ND & ND & $<0.1$ & 0.016 \\
\hline WL23 & 20.8 & 0.927 & 41.70 & 86.60 & 0.000 & 0.018 & ND & ND & $<0.1$ & 0.000 \\
\hline WL25 & 48.0 & 1.075 & 27.60 & 46.60 & 0.004 & 0.29 & ND & ND & $<0.1$ & 0.026 \\
\hline
\end{tabular}

ND - NOT DETECTED. $\mathrm{Cd}$ and $\mathrm{Pb}$ are below detection limits.

Table 3. The result of chemical characterstics (anions) of surface water and groundwater around okemesi

\begin{tabular}{lllllllll}
\hline S/N & SAMPLE ID & SAR & $\begin{array}{l}\mathbf{S i O}_{2} \\
(\mathbf{m g} / \mathbf{l})\end{array}$ & $\begin{array}{l}\mathbf{H C O}_{3}{ }^{-} \\
(\mathbf{m g} / \mathbf{l})\end{array}$ & $\begin{array}{l}\mathbf{C l}^{-} \\
(\mathbf{m g} / \mathbf{l})\end{array}$ & $\begin{array}{l}\mathbf{S O}_{4}{ }^{2-} \\
(\mathbf{m g} / \mathbf{l})\end{array}$ & $\begin{array}{l}\mathbf{N O}_{3}{ }^{2-} \\
(\mathbf{m g} / \mathbf{l})\end{array}$ & $\begin{array}{l}\mathbf{P O}_{4}{ }^{3-} \\
(\mathbf{m g} / \mathbf{l})\end{array}$ \\
\hline 1 & RL1 & 2.38 & 9.0 & 12.2 & 12.5 & $<35.0$ & 0.000 & 0.011 \\
2 & WL2 & 1.68 & 9.0 & 24.4 & 11.5 & $<35.0$ & 0.000 & 0.009 \\
3 & RL3 & 2.74 & 4.0 & 24.4 & 12.0 & $<35.0$ & 0.000 & 0.014 \\
4 & WL4 & 1.97 & $<4.0$ & 6.10 & 10.5 & $<35.0$ & 0.000 & 0.020 \\
5 & RL5 & 4.39 & 10 & 42.7 & 19.0 & 38.0 & 0.000 & 0.016 \\
6 & WL6 & 6.96 & 4.0 & 12.2 & 29.5 & $<35.0$ & 0.000 & 0.019 \\
7 & RL8 & 2.89 & 4.0 & 18.3 & 5.00 & $<35.0$ & 0.000 & 0.012 \\
8 & SPL9 & 0.80 & 8.0 & 6.10 & 9.50 & $<35.0$ & 0.000 & 0.005 \\
9 & WL10 & 0.88 & 8.0 & 6.10 & 15.0 & $<35.0$ & 0.000 & 0.028 \\
10 & BL11 & 1.57 & 8.0 & 30.5 & 12.5 & $<35.0$ & 0.000 & 0.016 \\
11 & BL12 & 2.08 & 6.0 & 12.2 & 8.50 & $<35.0$ & 0.000 & 0.018 \\
12 & WL13 & 1.26 & 8.0 & 18.3 & 18.0 & $<35.0$ & 0.000 & 0.030 \\
13 & SPL14 & 4.58 & 8.0 & 6.10 & 10.5 & $<35.0$ & 0.000 & 0.020 \\
14 & WL15 & 3.27 & 8.0 & 12.2 & 20.0 & $<35.0$ & 0.000 & 0.014 \\
15 & RL16 & 3.04 & 4.0 & 24.4 & 18.5 & $<35.0$ & 0.000 & 0.019 \\
16 & BL17 & 3.92 & 8.0 & 36.6 & 67.0 & 70.0 & 0.000 & 0.020 \\
17 & BL19 & 12.22 & 6.0 & 24.4 & 109.0 & $<35.0$ & 0.000 & 0.015 \\
18 & WL20 & 3.96 & 4.0 & 79.3 & 39.5 & $<35.0$ & 0.000 & 0.016 \\
19 & WL21 & 2.88 & 8.0 & 12.2 & 16.5 & 76.0 & 0.000 & 0.020 \\
20 & WL23 & 8.95 & 4.0 & 30.5 & 51.0 & 53.0 & 0.000 & 0.015 \\
21 & WL25 & 3.94 & $<4.0$ & 54.9 & 16.2 & 38.0 & 0.000 & 0.012 \\
\hline & & & & & & & &
\end{tabular}


Table 4. The result of the bacteriological analysis around Okemesi

\begin{tabular}{lllllll}
\hline S/N & SAMPLE & $\begin{array}{l}\text { CHLORINE } \\
\text { ID }\end{array}$ & $\begin{array}{l}\text { COLONY } \\
\text { COUNT } \\
\text { (MPN) }\end{array}$ & $\begin{array}{l}\text { MPN } \\
\text { COLIFORM } \\
\text { ORGANISM }\end{array}$ & LOCATION & ROCK TYPE \\
\hline 1 & RL1 & 0 & 30 & 30 & Ada & Quartz schist \\
2 & WL2 & 0 & 80 & 80 & Oke Onire 1 & Mica schist \\
3 & RL3 & 0 & 40 & 40 & Aba Paanu & Quartzite \\
4 & WL4 & 0 & 10 & 10 & Aba Paanu 2 & Quartzite \\
5 & RL5 & 0 & 20 & 20 & Ajindo/Koro & Amphibolite \\
6 & WL6 & 0 & 20 & 20 & Oke Onire 2 & Mica schist \\
7 & RL8 & 0 & 20 & 20 & Oke Onire 3 & Quartzite \\
8 & SPL9 & 0 & 10 & 10 & Ikanwo & Quartzite \\
9 & WL10 & 0 & 40 & 40 & Oke Oruro & Mica schist \\
10 & BL11 & 0 & 20 & 20 & Oke Onire 4 & Quartzite \\
11 & BL12 & 0 & 10 & 10 & Oke Onire 5 & Quartzite \\
12 & WL13 & 0 & 20 & 20 & Ile Obanla & Banded gneiss \\
13 & SPL14 & 0 & 5 & Omioko & Quartz schist \\
14 & WL15 & 0 & 10 & 10 & Okemobi & Quartz schist \\
15 & RL16 & 0 & 20 & 20 & Eleyinmi & Quartz schist \\
16 & BL17 & 0 & 10 & 10 & Iro & Mica schist \\
17 & BL19 & 0 & 20 & 20 & Odofin & Banded gneiss \\
18 & WL20 & 0 & 40 & 40 & Obanurin & Banded gneiss \\
19 & WL21 & 0 & 30 & 30 & Okenoran & Mica schist \\
20 & WL23 & 0 & 10 & 10 & Obanla & Quartz schist \\
21 & WL25 & 0 & 30 & 30 & Odoobi & Mica schist \\
\hline & & & & &
\end{tabular}

The summary of the various parameters, their mean values as compared to the values of WHO (2004) standards is shown in table 5, while the result of the bacteriological analysis as compared with World Health Organization (W.H.O) 2004 Bacteriological Standard for Drinking Water is in Table 6.

The mean concentration of the cations is in the order $\mathrm{Ca}^{2+}>\mathrm{K}^{+}>\mathrm{Na}^{+}>\mathrm{Mg}^{2+}$ and the mean concentration of anions is in the order $\mathrm{SO}_{4}{ }^{2-}>\mathrm{Cl}^{-}>\mathrm{HCO}_{3}>$ Silica $>\mathrm{PO}_{4}{ }^{3-}>\mathrm{NO}_{3}{ }^{2-}$ while the trace metals mean concentration is in the order of $\mathrm{Fe}>\mathrm{Cu}>\mathrm{Zn}>\mathrm{Ni}>\mathrm{Cd}>\mathrm{Pb}$ and that of coliform mean concentration is 24ppm. Although all the analyzed parameters have concentration values less than the World Health Organization (WHO, 2004) recommended limits, $\mathrm{K}^{+}$in locations WL6 (16.20mg/l), WL13 (25.40mg/l), BL17 (23.6mg/l), BL19 (108.3mg/l), WL20 (48.2mg/l), WL23 $(86.6 \mathrm{mg} / \mathrm{l})$, and WL25 $(46.6 \mathrm{mg} / \mathrm{l})$ were observed to have the concentration value above WHO standard recommended limit (Table 5). These are all groundwater. Generally the concentration of calcium is much higher in the deep wells than the surface waters. for instance, sample BL17, BL19,WL20, and WL25 have calcium concentration of $72.8 \mathrm{mg} / 1,36.8 \mathrm{mg} / 1,64.0 \mathrm{mg} / 1$, and $48.0 \mathrm{mg} / 1$ respectively while RL1, RL3, SPL9 and SPL14 have calcium concentration $4.8 \mathrm{mg} / 1,9.6 \mathrm{mg} / 1,4.0 \mathrm{mg} / \mathrm{l}$, and $1.6 \mathrm{mg} / \mathrm{l}$ respectively. 
Table 5. Summary of the physical and chemical parameters of waters around okemesi compared with WHO (2004) standard

\begin{tabular}{|c|c|c|c|c|c|c|c|}
\hline PARAMETERS & $\mathrm{N}$ & MINIMUM & MAXIMUM & MEAN & $\begin{array}{l}\text { STANDARD } \\
\text { DEVIATION }\end{array}$ & $\begin{array}{l}\text { HIGHEST } \\
\text { DESIRABLE } \\
\text { LEVEL (WHO) }\end{array}$ & $\begin{array}{l}\text { MAXIMUM } \\
\text { PERMISSIBLE } \\
\text { LEVEL (WHO) }\end{array}$ \\
\hline $\begin{array}{l}\text { ELEVATION } \\
(\mathrm{m})\end{array}$ & 21 & 318.00 & 484.001 & 410.33 & $\mathrm{NC}$ & & \\
\hline TDS (mg/l) & 21 & 18.75 & 790.50 & 209.38 & 229.227 & 500 & 1000 \\
\hline $\mathrm{EC}(\mu \mathrm{S} / \mathrm{cm})$ & 21 & 25 & 1054 & 284.59 & 302.015 & 1000 & 1400 \\
\hline $\mathrm{Ph}$ & 21 & 5.4 & 7.4 & 6.32 & 0.532 & $7.0-8.5$ & $6.5-9.2$ \\
\hline TEMP $\left({ }^{\circ} \mathrm{C}\right)$ & 21 & 24.8 & 29.5 & 26.70 & 1.312 & variable & variable \\
\hline TURB (N.T.U.) & 21 & 2.2 & 40.5 & 11.85 & $\mathrm{NC}$ & 5 & $\mathrm{NM}$ \\
\hline $\mathrm{TH}(\mathrm{mg} / \mathrm{l})$ & 21 & 10 & 274.00 & 80.48 & 82.197 & $\mathrm{NM}$ & $\mathrm{NM}$ \\
\hline $\mathrm{TA}(\mathrm{mg} / \mathrm{l})$ & 21 & 18 & 274.00 & 72.10 & 67.292 & NM & NM \\
\hline $\mathrm{DO}(\mathrm{mg} / \mathrm{l})$ & 21 & 1.8 & 7.00 & 4.57 & $\mathrm{NC}$ & NM & $\mathrm{NM}$ \\
\hline $\mathrm{CD}(\mathrm{mg} / \mathrm{l})$ & 21 & 1.56 & 4.75 & 2.30 & $\mathrm{NC}$ & $\mathrm{NM}$ & $\mathrm{NM}$ \\
\hline MPN (PPM) & 21 & 5 & 80 & 23.57 & $\mathrm{NC}$ & NM & $\mathrm{NM}$ \\
\hline SAR & 21 & 0.8 & 12.22 & 3.64 & $\mathrm{NC}$ & NM & NM \\
\hline $\mathrm{Ca}^{2+}(\mathrm{mg} / \mathrm{l})$ & 21 & 1.6 & 72.80 & 19.73 & 20.351 & 75 & 200 \\
\hline $\mathrm{Mg}^{2+}(\mathrm{mg} / \mathrm{l})$ & 21 & 0.00 & 4.39 & 0.84 & 0.998 & 39 & 150 \\
\hline $\mathrm{Na}^{+}(\mathrm{mg} / \mathrm{l})$ & 21 & 1.63 & 75.0 & 16.56 & 17.958 & 150 & 200 \\
\hline $\mathrm{K}^{+}(\mathrm{mg} / \mathrm{l})$ & 21 & 0.00 & 108.30 & 19.43 & 29.614 & 10 & 15 \\
\hline $\mathrm{SiO}_{2}(\mathrm{mg} / \mathrm{l})$ & 21 & 4.0 & 10.00 & 6.74 & 2.137 & 20 & $\mathrm{NM}$ \\
\hline $\mathrm{HCO}_{3}{ }^{-}(\mathrm{mg} / \mathrm{l})$ & 21 & 6.1 & 79.30 & 23.53 & 18.227 & 500 & 1000 \\
\hline $\mathrm{Cl}^{-}(\mathrm{mg} / \mathrm{l})$ & 21 & 5.0 & 109 & 24.37 & 24.614 & 200 & 500 \\
\hline $\mathrm{SO}_{4}^{2-}(\mathrm{mg} / \mathrm{l})$ & 21 & 38 & 76 & 55 & 11.773 & 150 & 250 \\
\hline $\mathrm{NO}_{3}{ }^{2-}(\mathrm{mg} / \mathrm{l})$ & 21 & ND & 0.00 & 0.00 & $\mathrm{NC}$ & 20 & 45 \\
\hline $\mathrm{PO}_{4}{ }^{3-}(\mathrm{mg} / \mathrm{l})$ & 21 & 0.005 & 0.03 & 0.02 & 0.006 & $\mathrm{NM}$ & $\mathrm{NM}$ \\
\hline $\mathrm{Cu}(\mathrm{mg} / \mathrm{l})$ & 21 & ND & 0.391 & 0.04 & 0.082 & 1.0 & 1.5 \\
\hline $\mathrm{Zn}(\mathrm{mg} / \mathrm{l})$ & 21 & ND & 0.29 & 0.02 & 0.064 & 0.2 & 5.0 \\
\hline $\mathrm{Cd}(\mathrm{mg} / \mathrm{l})$ & 21 & ND & 0.00 & 0.00 & $\mathrm{NC}$ & 0 & 0.005 \\
\hline $\mathrm{Pb}(\mathrm{mg} / \mathrm{l})$ & 21 & ND & 0.00 & 0.00 & $\mathrm{NC}$ & 0 & 0.05 \\
\hline $\mathrm{Fe}(\mathrm{mg} / \mathrm{l})$ & 21 & 0.10 & 0.10 & 0.10 & $\mathrm{NC}$ & $0 . .3$ & 1.0 \\
\hline $\mathrm{Ni}(\mathrm{mg} / \mathrm{l})$ & 21 & 0.00 & 0.043 & 0.016 & 0.012 & 0 & 0.02 \\
\hline
\end{tabular}

NC - NOT CALCULATED NM - NOT MENTIONED

Table 6. Bacteriological standard for drinking water compared with those around Okemesi area (WHO 2004)

\begin{tabular}{|c|c|c|c|}
\hline $\mathrm{S} / \mathrm{N}$ & CLASSIFICATION & $\begin{array}{l}\text { MPN/100ml } \\
\text { COLIFORM } \\
\text { BACTERIA }\end{array}$ & $\begin{array}{l}\text { SUMMARY FOR } \\
\text { OKEMESI }\end{array}$ \\
\hline 1 & Bacterial quality applicable to disinfection only & $0-50$ & 20 \\
\hline 2 & $\begin{array}{l}\text { Bacterial quality requiring convectional methods of } \\
\text { treatment (coagulation, filteration and di-infection) }\end{array}$ & $50-5,000$ & 1 \\
\hline 3 & Heavy pollutant requiring extensive type of treatment & $5,000-50,000$ & - \\
\hline 4 & $\begin{array}{l}\text { Very heavy pollution, unacceptable unless special } \\
\text { treatments designed for such water is used. }\end{array}$ & Above 50,000 & - \\
\hline
\end{tabular}


In this study, the highest concentration of $\mathrm{Ca}^{2+}$ is found in borehole location BL17 at Iro, Okemesi having value of $72.8 \mathrm{mg} / \mathrm{l}$. This may be attributed to dissolution of the water with the weathered calc-plagioclase feldspar, amphibole and pyroxene of the aquiferous zone by the groundwater. However, $\mathrm{Mg}^{2+}$ concentration in all the location is very low with mean value of $0.84 \mathrm{mg} / 1$ when compared to WHO highest desirable level of $39 \mathrm{mg} / \mathrm{l}$ for drinking water and could be attributed to generally lower dissolution of magnesium in water. Its values range from $0.146 \mathrm{mg} / \mathrm{l}$ to $2.245 \mathrm{mg} / \mathrm{l}$ in most places except in location RL5 at river Koro where the concentration is $4.390 \mathrm{mg} / \mathrm{l}$. This river also has the highest concentration of $\mathrm{Ca}^{2+}$ of $28.8 \mathrm{mg} / 1$ for surface water. This may have incorporated the elemental component of the amphibolite in this area that the river passes through (Table 7).

Table 7. The statistical summary of average elemental composition of water sample control by rock type around Okemesi compared with WHO standard

\begin{tabular}{|c|c|c|c|c|c|c|}
\hline $\begin{array}{l}\text { ELEMENTS } \\
(\mathrm{mg} / \mathrm{l})\end{array}$ & $\begin{array}{l}\text { AVERAGE } \\
\text { FROM THE } \\
\text { WATER } \\
\text { SAMPLE OF } \\
\begin{array}{l}\text { AMPHIBOLITE } \\
(\mathrm{mg} / \mathrm{l})\end{array}\end{array}$ & $\begin{array}{l}\text { AVERAGE } \\
\text { FROM } \\
\text { THE } \\
\text { WATER } \\
\text { SAMPLE } \\
\text { OF } \\
\text { BANDED } \\
\text { GNEISS } \\
(\mathrm{mg} / \mathrm{l})\end{array}$ & $\begin{array}{l}\text { AVERAGE } \\
\text { FROM } \\
\text { THE } \\
\text { WATEROF } \\
\text { MICA } \\
\text { SCHIST } \\
(\mathrm{mg} / \mathrm{l})\end{array}$ & $\begin{array}{l}\text { AVERAGE } \\
\text { FROM } \\
\text { THE } \\
\text { WATER } \\
\text { SAMPLE } \\
\text { OF } \\
\text { QUARTZ } \\
\text { SCHIST } \\
(\mathrm{mg} / \mathrm{l})\end{array}$ & $\begin{array}{l}\text { AVERAGE } \\
\text { FROM } \\
\text { WATER } \\
\text { SAMPLE OF } \\
\text { THE } \\
\text { QUARTZITE } \\
(\mathrm{mg} / \mathrm{l})\end{array}$ & $\begin{array}{l}\text { W. H. O } \\
\text { STANDARD } \\
2004 \\
(\mathrm{mg} / \mathrm{l})\end{array}$ \\
\hline $\mathrm{Ca}^{2+}$ & 28.8 & 37.33 & 29.07 & 12.80 & 10.13 & 75 \\
\hline $\mathrm{Mg}^{2+}$ & 4.39 & 1.25 & 0.80 & 0.46 & 0.30 & 39 \\
\hline $\mathrm{Na}^{+}$ & 25.3 & 37.18 & 16.94 & 15.85 & 4.93 & 150 \\
\hline $\mathrm{K}^{+}$ & 1.43 & 58.97 & 17.01 & 19.598 & 4.11 & 10 \\
\hline $\mathrm{SiO}_{2}$ & 10.0 & 6.0 & 7.17 & 6.60 & 5.70 & 20 \\
\hline $\mathrm{HCO}_{3}^{-}$ & 42.7 & 40.67 & 24.73 & 17.08 & 16.27 & 500 \\
\hline $\mathrm{Cl}^{-}$ & 19.0 & 55.5 & 25.95 & 33.8 & 9.67 & 200 \\
\hline $\mathrm{SO}_{4}{ }^{2-}$ & 38.0 & 35.0 & 48.17 & 38.6 & 35.0 & 150 \\
\hline $\mathrm{NO}_{3}{ }^{2-}$ & 0.00 & 0.00 & 0.00 & 0.00 & 0.00 & 20 \\
\hline $\mathrm{PO}_{4}^{3-}$ & 0.016 & 0.02 & 0.02 & 0.02 & 0.01 & \\
\hline $\mathrm{Cu}$ & 0.018 & 0.02 & 0.03 & 0.03 & 0.01 & 1.0 \\
\hline $\mathrm{Zn}$ & 0.000 & 0.01 & 0.06 & 0.01 & 0.02 & 0.2 \\
\hline $\mathrm{Cd}$ & 0.000 & 0.00 & 0.00 & 0.00 & 0.00 & 0.005 \\
\hline $\mathrm{Pb}$ & 0.000 & 0.00 & 0.00 & 0.00 & 0.00 & 0.05 \\
\hline $\mathrm{Fe}$ & 0.1 & 0.10 & 0.10 & 0.10 & 0.00 & 0.30 \\
\hline $\mathrm{Ni}$ & 0.006 & 0.03 & 0.02 & 0.01 & 0.02 & 0.02 \\
\hline
\end{tabular}

Generally, the concentration of $\mathrm{K}^{+}$and $\mathrm{Na}^{+}$varies for surface and groundwater. The highest $\mathrm{K}^{+}$and $\mathrm{Na}^{+}$concentration value of $108.3 \mathrm{mg} / \mathrm{l}$ and $75 \mathrm{mg} / \mathrm{l}$ respectively is found in borehole BL19 at Odofin while the lowest values of 0.00 and 1.63 for the two cations are found respectively at Ikanwo hills at location SPL9. This can be attributed to difference in the rock types and elevations of these two locations. While Odofin rock is banded gneiss with elevation of $414 \mathrm{~m}$, Ikanwo hills is quartzitic in composition with elevation of $484 \mathrm{~m}$ above the sea level (Table 7). Hence, the borehole must have incorporated some minerals such as albite and orthoclase in the gneiss. The chloride concentration seems to have relationship with sodium and potassium in the area of investigation. This is because high concentration of chloride ion is found in area of high $\mathrm{K}^{+}$and $\mathrm{Na}^{+}$. For example, sample BL19 with abnormal $\mathrm{Cl}^{-}, \mathrm{K}^{+}$and $\mathrm{Na}^{+}$ concentration values of $109 \mathrm{mg} / \mathrm{l}, 108.3 \mathrm{mg} / \mathrm{l}$, and $75 \mathrm{mg} / \mathrm{l}$, may have important geological control.

The source of $\mathrm{HCO}_{3}$ with mean concentration of $23.53 \mathrm{mg} / \mathrm{l}$ can be attributed to dissolution of carbon dioxide of the air and organic matter. The lower concentration or absence of $\mathrm{SO}_{4}{ }^{2-}, \mathrm{PO}_{4}{ }^{3-}, \mathrm{NO}_{3}{ }^{2-}, \mathrm{Fe}, \mathrm{Cu}, \mathrm{Zn}, \mathrm{Cd}$ and $\mathrm{Pb}$ in all the locations could be attributed to absent of industrial discharges, sewage discharge, landfill leachates and ore 
bodies. However, Ni concentration value is slightly above the maximum recommended value by WHO in 3 wells in location WL20, WL15 and WL4. Elevated trace metals concentrations in soils and shallow groundwater systems have been attributed to anthropogenic sources through agricultural practices (Abimbola et al., 1999: Mapanda et al., 2005; Tijani et al., 2009; Tijani, 2010) the possibility of geogenic contribution is high considering the presence of a basic rock unit associated with the gneisses in the area. The relatively higher electrical conductivity with a range of $50 \mu \mathrm{s} / \mathrm{cm}$ to $1054 \mu \mathrm{s} / \mathrm{cm}$ in the groundwater compared to a range of $25 \mu \mathrm{s} / \mathrm{cm}$ to $535 \mu \mathrm{s} / \mathrm{cm}$ in the surface water may also be due to increased water rock reaction and dissolution of solid components of the bed rock. However majority of the values are still within those for unpolluted waters

Statistical correlation using product moment coefficient and scattered plot indicates positive correlation between some pairs of parameters (Tables $8 \& 9$ ) and. Correlation studies show that calcium has positive correlation with cations: $\mathrm{Mg}(\mathrm{r}=0.584), \mathrm{Na}(\mathrm{r}=0.633), \mathrm{K}(\mathrm{r}=0.481)$ and some heavy metals: $\mathrm{Zn}(0.342)$ and $\mathrm{Ni}(0.505)$ indicating common source but show negative correlation with $\mathrm{Cu}(\mathrm{r}=-0.099)$ suggesting different source. Also, potassium correlates positively

Table 8. Correlation coefficients for the physico-chemical parameters around Okemesi

\begin{tabular}{|c|c|c|c|c|c|c|c|c|c|c|c|c|c|c|c|}
\hline & $\mathrm{Ca}$ & Mg & $\mathbf{N a}$ & $\mathbf{K}$ & $\mathrm{HCO}_{3}$ & Cl & $\mathrm{SO}_{4}$ & $\mathrm{SiO}_{2}$ & $\mathrm{PO}_{4}$ & $\mathbf{C u}$ & $\mathrm{Zn}$ & $\mathbf{N i}$ & TDS & EC & pH \\
\hline $\mathrm{Ca}$ & 1 & & & & & & & & & & & & & & \\
\hline Mg & .584 & 1 & & & & & & & & & & & & & \\
\hline $\mathbf{N a}$ & .633 & .531 & 1 & & & & & & & & & & & & \\
\hline $\mathbf{K}$ & .481 & .258 & .893 & 1 & & & & & & & & & & & \\
\hline $\mathrm{HCO}_{3}$ & .739 & .568 & .455 & .414 & 1 & & & & & & & & & & \\
\hline Cl & .588 & .407 & .930 & .832 & .293 & 1 & & & & & & & & & \\
\hline $\mathrm{SO}_{4}$ & .294 & .198 & .173 & .104 & .063 & .278 & 1 & & & & & & & & \\
\hline $\mathrm{SiO}_{2}$ & .005 & .251 & -.202 & -.327 & -.168 & -.140 & .142 & 1 & & & & & & & \\
\hline PO4 & .031 & -.035 & -.090 & -.037 & -.152 & .049 & .157 & -.009 & 1 & & & & & & \\
\hline $\mathrm{Cu}$ & -.099 & -.107 & -.190 & -.167 & -.252 & -.184 & -.064 & -.220 & .151 & 1 & & & & & \\
\hline $\mathrm{Zn}$ & .342 & .049 & .188 & .258 & .315 & -.011 & -.016 & -.271 & -.108 & .138 & 1 & & & & \\
\hline $\mathbf{N i}$ & .505 & .121 & .258 & .192 & .273 & .257 & .032 & -.157 & .020 & .486 & .318 & 1 & & & \\
\hline TDS & .816 & .671 & .933 & .815 & .618 & .889 & .288 & -.070 & .013 & -.216 & .223 & .301 & 1 & & \\
\hline EC & .817 & .668 & .936 & .817 & .625 & .891 & .284 & -.092 & .006 & -.224 & .221 & .287 & .997 & 1 & \\
\hline pH & -.025 & .121 & .001 & .022 & .320 & -.161 & .008 & -.091 & -.232 & -.174 & .284 & -.140 & -.019 & .015 & 1 \\
\hline
\end{tabular}

Table 9. Correlations between some of the hydrochemical parameters around Okemesi

\begin{tabular}{cc}
\hline Variable & Correlation Coefficient \\
\hline $\mathrm{Mg}^{2+}$ and $\mathrm{Ca}^{2+}$ & 0.58 \\
$\mathrm{Mg}^{2+}$ and $\mathrm{EC}$ & 0.69 \\
$\mathrm{~K}^{+}$and $\mathrm{Na}^{+}$ & 0.89 \\
$\mathrm{~K}^{+}$and $\mathrm{EC}$ & 0.82 \\
$\mathrm{Na}^{+}$and $\mathrm{EC}$ & 0.94 \\
$\mathrm{TDS}$ and $\mathrm{Ca}^{2+}$ & 0.82 \\
$\mathrm{TDS}$ and $\mathrm{Mg}^{2+}$ & 0.67 \\
$\mathrm{Mg}^{2+}$ and $\mathrm{TH}^{-}$ & 0.87 \\
$\mathrm{TDS}$ and $\mathrm{HCO}_{3}^{-}$ & 0.62 \\
$\mathrm{Na}^{+}$and $\mathrm{Cl}^{-}$ & 0.93 \\
$\mathrm{Mg}^{2+}$ and $\mathrm{HCO}_{3}^{-}$ & 0.57 \\
$\mathrm{Na}^{+}$and $\mathrm{Ca}^{2+}$ & 0.63 \\
$\mathrm{~K}^{+}$and $\mathrm{Cl}^{-}$ & 0.83 \\
$\mathrm{Ni}$ and $\mathrm{Cu}^{2+}$ & 0.49 \\
$\mathrm{Ca}^{2+}$ and $\mathrm{Ni}$ & 0.51 \\
\hline
\end{tabular}


with $\mathrm{Na}(\mathrm{r}=0.893)$, chloride $(\mathrm{r}=0.832)$ and Carbonate $(\mathrm{r}=0.414)$ indicating the same source and show negative correlation with silica $(r=-0.327)$, phosphate $(r=-0.037)$ and copper $(r=-0.167)$. While correlation coefficient of sodium with carbonate $(\mathrm{r}=0.455)$, chloride $(\mathrm{r}=0.930)$, sulphate $(\mathrm{r}=0.173)$, Zinc $(\mathrm{r}=0.188)$ and Nickel $(\mathrm{r}=0.258)$ are positive, its correlation with silica $(\mathrm{r}=-0.202)$, phosphate $(\mathrm{r}=-0.090)$ and copper $(\mathrm{r}=-0.190)$ is negative. However, $\mathrm{Ni}$ has a weak positive correlation to all the ions; Calcium $(\mathrm{r}=0.505)$, magnesium $(\mathrm{r}=0.121)$, sodium $(\mathrm{r}=0.258)$, potassium $(\mathrm{r}=0.192)$, carbonate $(\mathrm{r}=0.273)$, chloride $(\mathrm{r}=0.257)$, sulphate $(\mathrm{r}=0.032)$, phosphate $(\mathrm{r}=$ $0.020)$, and copper $(\mathrm{r}=0.486)$, except silica $(\mathrm{r}=-0.070)$. There are relatively very strong correlation between total hardness and total alkalinity $(\mathrm{r}=0.835)$, total dissolved solid and electrical conductivity $(\mathrm{r}=0.997)$, total hardness and electrical conductivity $(\mathrm{r}=0.849)$, calcium and total hardness $(\mathrm{r}=0.893)$, magnesium and total hardness $(\mathrm{r}=0.869)$.

\subsection{Water Characterization}

The plots of chemical parameters were carried out using trilinear Piper (Piper, 1944) and Scholler semi-log (Scholler, 1962) diagrams (Figures $3 \& 4$ ), to determine hydrochemical facies of the analyzed water sample collected around Okemesi. The Piper-Hill diagram is used to infer hydro-geochemical facies. These diagrams reveal the analogies, dissimilarities and different types of waters in the study area, which are identified and listed in Table 10. The concept of hydrochemical facies was developed in order to understand and identify the water composition in different classes. (Sadashivaiah et al., 2008). This water characterization by Piper's diagram has revealed 2 important water facies around Okemesi and these are $\mathrm{Ca}-(\mathrm{Mg})-(\mathrm{Cl})-\mathrm{SO}_{4}$ and $\mathrm{Na}-(\mathrm{k})-\mathrm{Cl}-(\mathrm{SO})$ water facies.

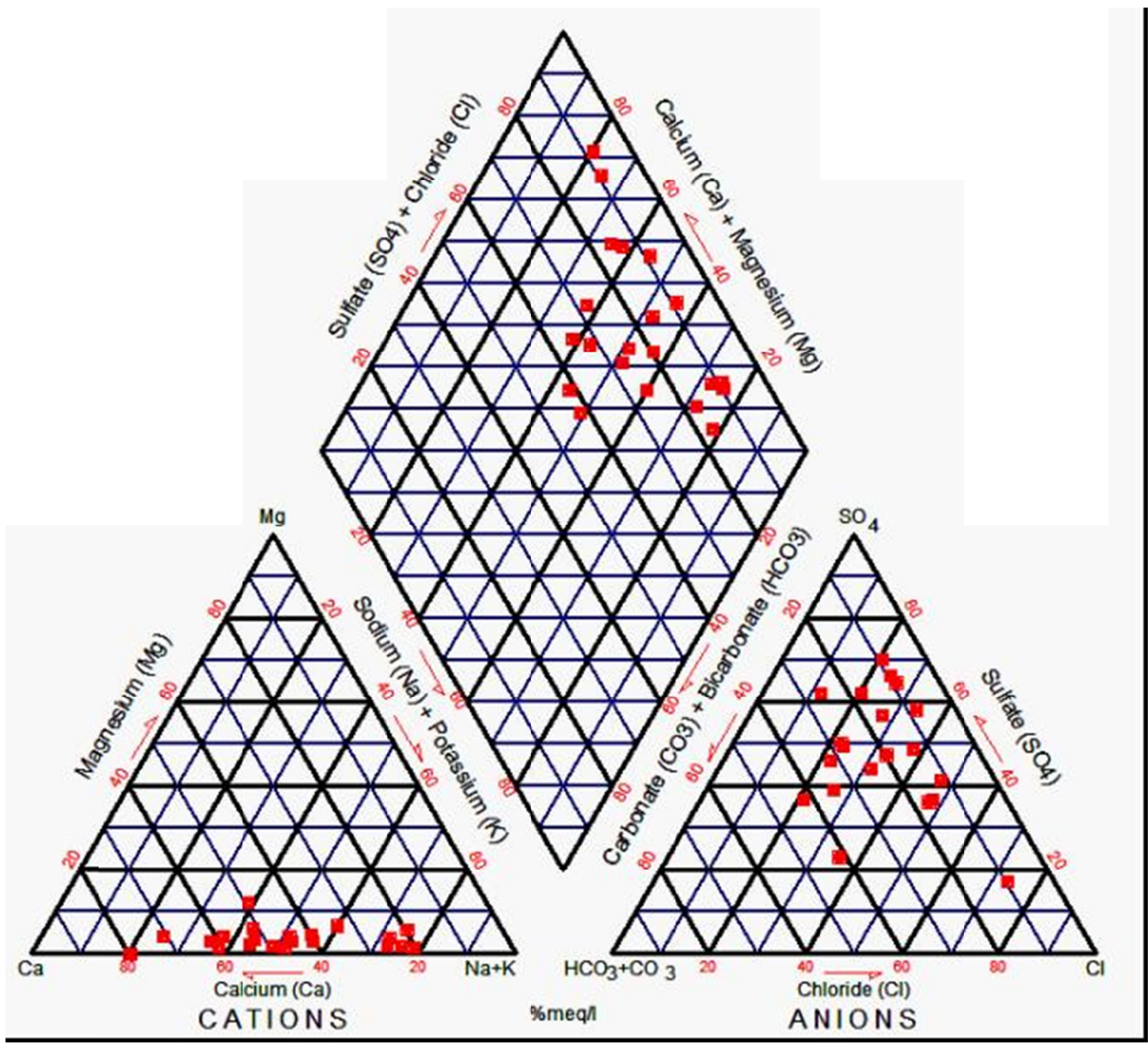

Figure 3. Piper Trilinear Diagram (1944) of hydrochemical analysis result around Okemesi 
Table 10. Characterization of groundwater and surface water around Okemesi on the basis of Piper tri-linear diagram

\begin{tabular}{|c|c|c|}
\hline $\begin{array}{l}\text { Subdivision of the } \\
\text { diamond }\end{array}$ & $\begin{array}{l}\text { Characteristics of corresponding subdivisions of } \\
\text { diamond-shaped fields }\end{array}$ & $\begin{array}{l}\text { Percentage of samples in this } \\
\text { category }\end{array}$ \\
\hline 1 & Alkaline earth $(\mathrm{Ca}+\mathrm{Mg})$ Exceed alkali $(\mathrm{Na}+\mathrm{K})$ & 50 \\
\hline 2 & Alkali exceeds alkaline earths & 50 \\
\hline \multirow[t]{2}{*}{3} & Weak acids $(\mathrm{C} 03+\mathrm{HCO} 3)$ exceed & 0 \\
\hline & Strong acids $(\mathrm{SO} 4+\mathrm{Cl})$ & \\
\hline 4 & Strong acids exceeds weak acids & 100 \\
\hline 5 & Magnesium bicarbonate type & 0 \\
\hline 6 & Calcium-chloride type & 10 \\
\hline 7 & Sodium-chloride type & 52 \\
\hline 8 & Sodium-Bicarbonate type & 0 \\
\hline 9 & Mixed type (No cation - anion exceed 50\%) & 48 \\
\hline
\end{tabular}

$\mathrm{Ca}-(\mathrm{Mg})-(\mathrm{Cl})-\mathrm{SO}_{4}$ water facies constitute about $47.62 \%$ of the total water sample and is also referred to as normal alkaline earth with predominance of sulphate. This water suggests mixing process attributed to its interaction by dilution with weathered rock (Figure 5). It may also be due to anthropogenic input from inhabitant in the study area $. \mathrm{Na}-(\mathrm{k})-\left(\mathrm{SO}_{4}\right)-\mathrm{Cl}$ water facies constitute about $52.38 \%$ of the total surface and groundwater samples and as a result is predominant water type in the study area. This refers to as alkaline water with high concentration of sodium and potassium.

This water type is common in groundwater in the area and likely to have originated from the dissolution of weathered gneissic rocks and schist, that is geogenic in origin.

Table 11. Modified Wilcox Quality Classification (1995) of irrigation water compared with number of water samples collected around Okemesi

\begin{tabular}{|c|c|c|c|c|}
\hline $\begin{array}{l}\text { WATER } \\
\text { CLASS }\end{array}$ & $\begin{array}{l}\text { ELECTRICAL } \\
\text { CONDUCTIVITY (US/CM) }\end{array}$ & $\begin{array}{l}\text { SALINITY } \\
\text { HAZARD }\end{array}$ & SAR & $\begin{array}{l}\text { NUMBER OF SAMPLES } \\
\text { IN THIS CATEGORY }\end{array}$ \\
\hline EXCELLENT & $<250$ & LOW & $0-10$ & 20 \\
\hline GOOD & $250-750$ & MEDIUM & $10-18$ & 1 \\
\hline PERMISSIBLE & $750-2000$ & HIGH & $18-26$ & \\
\hline DOUBTFUL & $200-3000$ & VERY HIGH & $26-30$ & \\
\hline
\end{tabular}

Where,

$\mathrm{SAR}=\mathrm{Na}^{+} / \frac{\sqrt{\left(\mathrm{Ca}^{2+}+\mathrm{Mg}^{2+}\right)}}{2}$

Table 12. Classification of water hardness modified after Todd (1980), compared with number of water samples collected around Okemesi

\begin{tabular}{lcc}
\hline $\begin{array}{l}\text { TOTAL HARDNESS RANGE } \\
(\mathrm{mg} / \mathrm{L})\end{array}$ & WATER CLASS & $\begin{array}{c}\text { NUMBER OF WATER SAMPLES } \\
\text { IN THIS CATEGORY }\end{array}$ \\
\hline $0-60$ & SOFT & 14 \\
$61-120$ & MODERATELY HARD & 2 \\
$121-180$ & HARD & 2 \\
$>180$ & VERY HARD & 3 \\
\hline
\end{tabular}


From Schoeller semi - log diagram (Figure 4), the water within the study area can be characterized as $\mathrm{Ca}-(\mathrm{Mg})$ $\mathrm{Na}-(\mathrm{K})-\left(\mathrm{SO}_{4}\right)-\mathrm{Cl}$ water type because it constitutes about $99 \%$ of the total water types in the area. This is similar to the Piper characterization and is due to high percentage of chloride and sulphide ions in the water body, which is higher when compared to other ions within the study area.

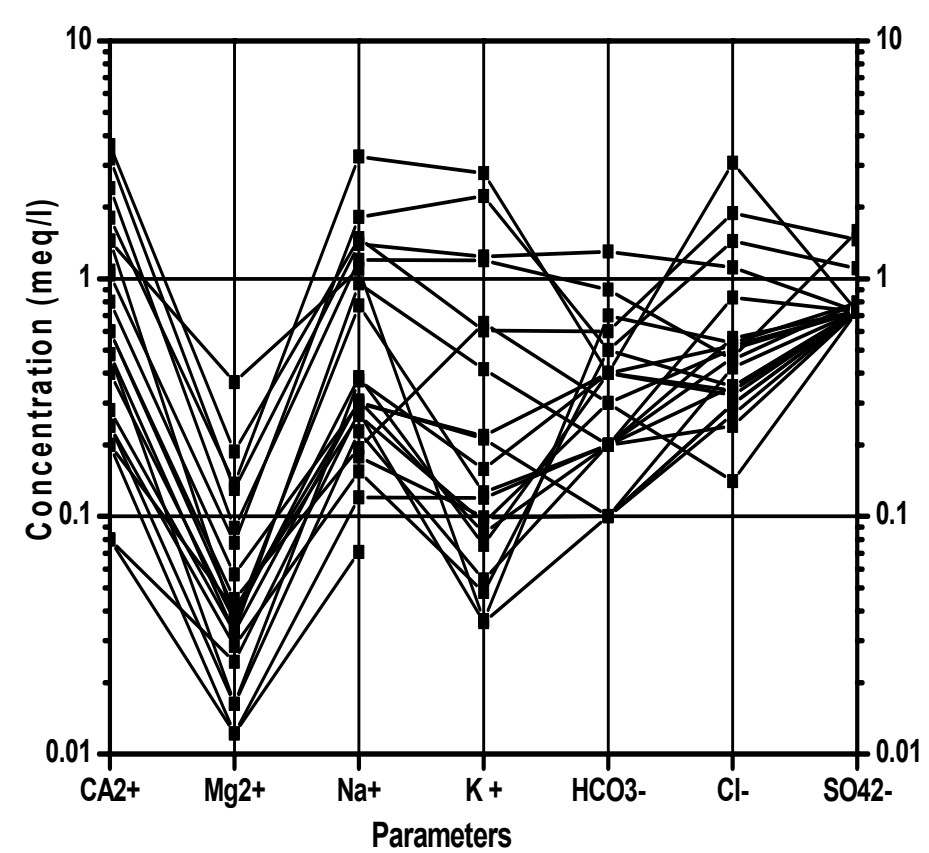

Figure 4. Scholler diagram showing chemical ions in the study area

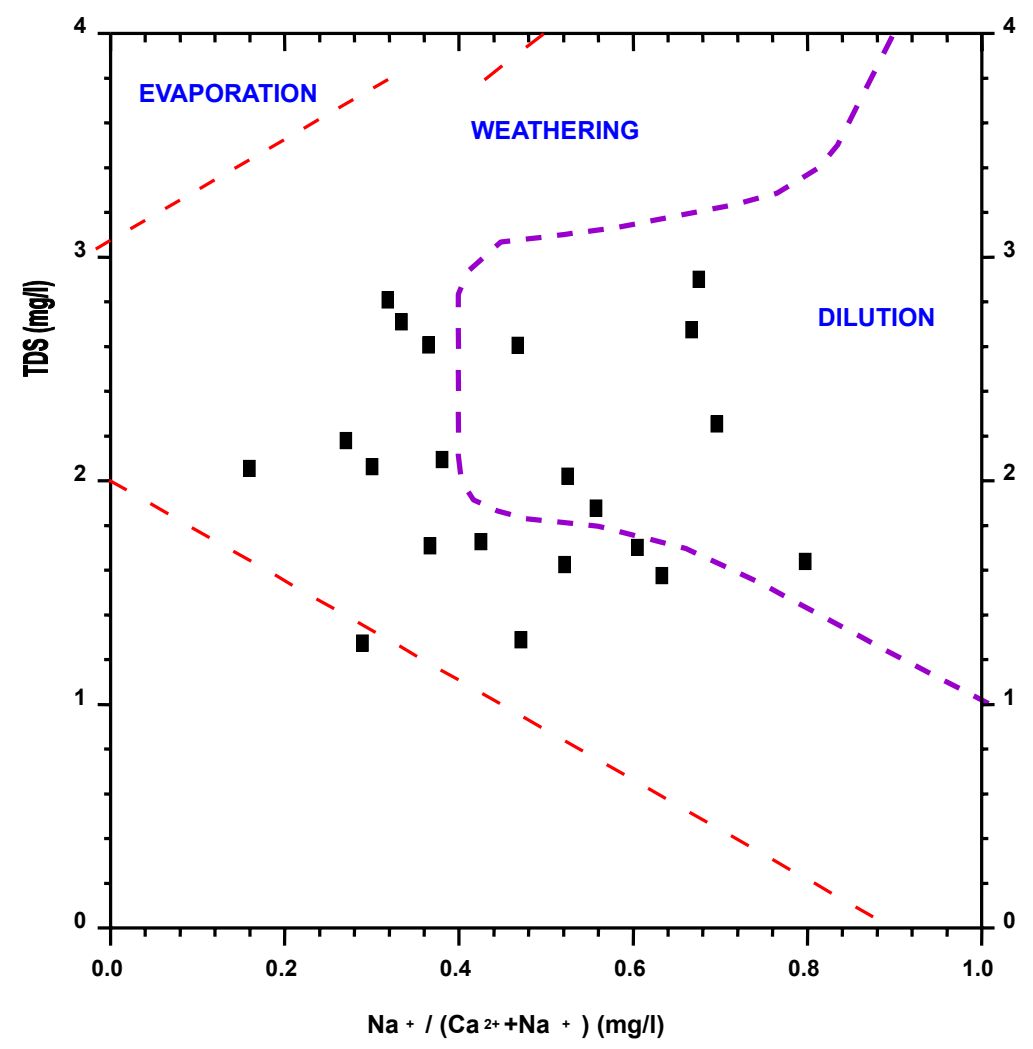

Figure 5. Modified Gibbs Diagram (1970) for the analysed water samples around Okemesi 
It is also apparent that the water character around Okemesi are influenced greatly by weathering and dissolution activities of the bedrock. The higher concentration of relevant ions associated with the groundwater as compared to surface water is attributed to longer resident time of the contact between the water and the rock bodies. This leads to greater dissolution, increases concentration of solutes and enhanced weathering more in subsurface water than in surface water (Figure 5).

\subsection{Water Quality and Usage}

The chemical character of any water source determines to some extent its quality and usability. Geology and human activities through mining, industrialization, disposal of waste are liable to cause deterioration in the quality of surface water and groundwater of any environment especially when the groundwater is closer to the earth surfce. (Abimbola et al., 2002). Also, the standard or criteria for portable drinking water according to Davis and DeWeist (1966) include the absence of objectionable tastes, odour, colour, and substance of adverse effects. A quantitative measure of these criteria is stipulated by WHO (2004). Water quality requirements for different purposes differ; hence standards have been developed to appraise water usability for the various purposes. (Olobaniyi et al., 2007).

The result of the hydrochemical analysis shows that the surface and groundwater within the study area are chemically portable as compared with the WHO standard. Also, the quantity of trace metals present in the different water samples is low compared with the WHO Standard, this may have resulted from the low $\mathrm{pH}$ where most natural groundwater are mobile and the mass occur as charged metal ion which reach equilibrium with the solid phase usually a metal-hydroxide, metal carbonate or metal sulphide (Domenico and Schwartz, 1998; Oke, 2010). However, few of the samples have nickel values exceeding the highest permissible level in places especially those water sample from banded gneiss rich in amphibolitic bands (Table 2 and 7). The result of the total coliform count also show that only one of the samples has concentration above the WHO standard which may be as a result of faecal contamination in the water supply (Table 4) and (Figure 6).

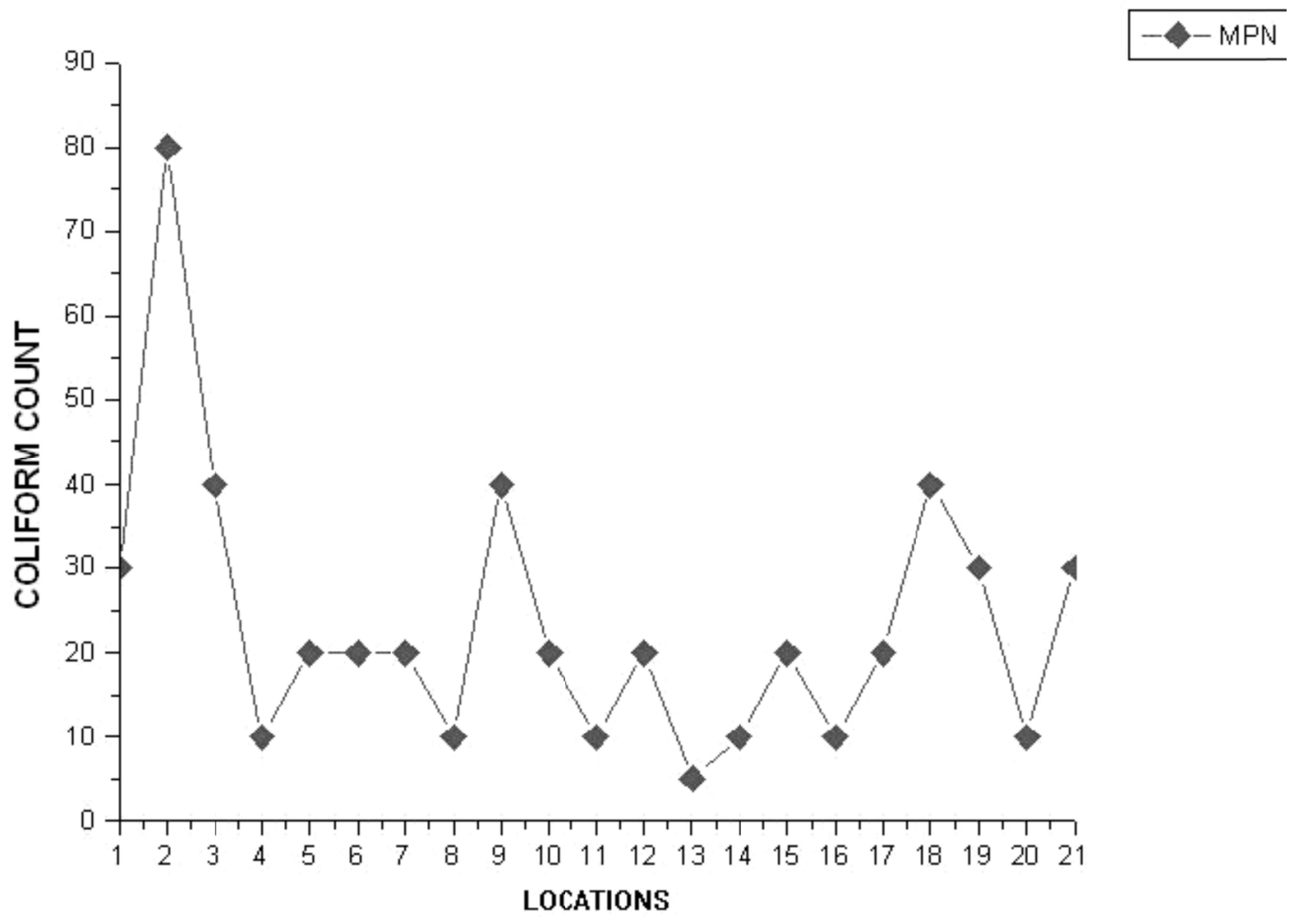

Figure 6. A plot of total coliform counts around Okemesi area against locations

\subsection{Irrigation Use}

The most important criteria for usage of water for irrigation purposes are total concentration of soluble salts, relative proportion of sodium to other principal cations, concentration of boron or other element that may be toxic, and under some condition, relationship of bicarbonate concentration with calcium and magnesium. These have been termed as the salinity hazard, sodium hazard, boron hazard and bicarbonate hazard (Wilcox, 1995). A better 
measure of the sodium hazard for irrigation is the SAR which is used to express reactions with the soil (Sadashivaiah et al., 2008). The classification of water samples from the study area with respect to SAR using Wilcox model is represented in Table 11. The SAR value of all the samples are found to be less than 10, and are classified as excellent for irrigation except in sample BL19 where SAR and electrical conductivity values are 12.22 and $1054 \mu \mathrm{s} / \mathrm{cm}$ respectively. This sample is within the permissible class and medium salinity hazard level. In general, all the water samples analysed are good for agricultural and irrigation purposes. However, other conditions necessary for plant growth such as amount of water, good soil type and favourable climate have to be met Also most of the samples $(67 \%)$ have total hardness within $0-60 \mathrm{mg} / \mathrm{l}$,implying they are soft while while the rest are moderately hard (Table 12)

\section{Conclusions}

This study showed that most of the hydrochemical parameters in the groundwater around Okemesi are within the WHO limits for drinking water, posing no health threat to consumers. However, the levels of potassium, especially in some groundwater samples especially those of WL6 (16.20mg/l), WL13 (25.4 mg/l), BL17 (108.3 mg/l), WL20 $(48.2 \mathrm{mg} / \mathrm{l}), \mathrm{WL} 23(86.6 \mathrm{mg} / \mathrm{l})$ and WL25 $(46.6 \mathrm{mg} / \mathrm{l})$, and, possibly, all the other areas such as WL4 $(0.043 \mathrm{mg} / \mathrm{l})$, WL15 (0.033) and WL20 (0.033) where nickel levels exceeded the critical value of $0.02 \mathrm{mg} / \mathrm{l}$, should be closely monitored. Geological processes such as weathering and dilution of the water with rocks in this area have great impact on the quality and characteristics of the groundwater. Also a shallow well at WL 2 from Oke-Onire is found to have total coli form count of $80 \mathrm{MPN}$, suggestive of heavy impact of human activity and is due to unnatural agents such as leakage of wastewaters and soak away.

Two major hydrochemical water facies were identified. These are $\mathrm{Ca}-(\mathrm{Mg})-(\mathrm{Cl})-\mathrm{SO}_{4}$ and $\mathrm{Na}-(\mathrm{K})-\mathrm{Cl}-\left(\mathrm{SO}_{4}\right)$ water types. Forty eight per cent of the water sources are mixed water type having either $\mathrm{HCO}_{3}, \mathrm{SO}_{4}{ }^{2-}$ or $\mathrm{Cl}^{-}$ions as the main anions predominating (Table 11).

Generally, the groundwater quality in this area is appropriate for drinking and agricultural purposes, except in few locations highlighted.

\section{Acknowledgements}

The authors appreciate the contribution of numerous colleagues and relations in the course of this study. The kings and chiefs of the study domains are specially acknowledged.

\section{References}

Abimbola, A. F., Ajibade, O. M., Odewande, A. A., Okunola, A. O., Laniyan, T. A., \& Kolawole, T. (2008). Hydrochemical characterization of water resources around the semi-urban area of Ijebu-Igbo southwestern, Nigeria. Water Resources. Journal of the Nigeria Association of Hydrogeologists, 18, 10-16.

Abimbola, A. F., Odukoya, A. M., \& Olatunjia, A. S. (2002). Influence of bedrock on the hydrogeochemical characteristics of bedrock of groundwater in Northern part of Ibadan metropolis. Water Resources. Journal of the Nigeria Association of Hydrogeologists, 13, 1-6.

Abimbola, A. F., Tijani, M. N., \& Nurudeen, S. I. (1999). Some aspects of groundwater quality in Abeokuta and its environs Southwestern Nigeria. Water Resources. Journal of the Nigeria Association of Hydrogeologists, 10, 6-11.

Acworth, R. I. (1999). The development of crystalline basement aquifers in a tropical environment. Quarterly Journal of Engineering Geology, 20, 603-613.

Adefemi, S. O., \& Awokunmi, E. E. (2010). Determination of physico-chemical parameters and heavy metals in water samples from Itaogbolu area of Ondo State, Nigeria. African Journal of Environmental Science and Technology, 4(3), 145-148p.

Adelana, S. M., \& Olasehinde, P. I. (2003). High nitrate in water supply in Nigeria: Implication for Human Health. Water Resources. Journal of Nigeria Association of Hydrogeologists, 4, 12-18.

Adeyemi, G. O., Adesile, A. O., \& Abayomi, O. B. (2003). Chemical characteristics of some well waters in Ikire, Southwestern Nigeria. Water Resources. Journal of the Nigeria Association of Hydrogeologists, 14, 12-18.

Adeyeye, E. L. (1994). Determination of heavy metals in Illisha Africana, associated water, soil sediments from some fish pond. International Journal of Environmental Study, 45, 231-240. http://dx.doi.org/10.1080/00207 239408710898

Amadi, P. A., Ofoegbu, C. O., \& Morrison, T. (1989). Hydrogeochemical assessment of groundwater quality in parts of the Niger Delta, Nigeria. Journal of Environmental Geology, 14(3), 195-202. 
Asaolu, S. S., Ipinmoroti, K. O., Adeyinowo, C. E., \& Olaofe, O. (1997). Interrelationship of heavy metals concentration in water, sediment as fish sample from Ondo state coastal area, Nigeria. African Journal of Science, 1, 55-61.

Ayodele, O. S., \& Odeyemi, I. B. (2010). Analysis of the lineaments extracted from LANDSAT image of the area around Okemesi, Southwestern Nigeria. Indian Journal of Science and Technology, 3(1), 31-36p.

Davis, S. N., \& De-Wiest, R. M. J. (1966). Hydrogeology. New York: John Wiley and sons.

Domenico, P. A., \& Schwartz, F. W. (1988). Physical and chemical hydrogeology. John Wiley and Son.

Fan, A. M., \& Steinberg, V. E. (1996). Health implications of nitrate and nitrate in drinking water: An update on methaeomoglobinaemia occurrence and reproductive and developmental toxicology, Regulatory toxicology and pharmacology. Academy Press International.

Gbodo, V. O., \& Ogunyemi, A. O. (1999). The quality and hydrogeochemistry of undergroundwater in Ago Iwoye/Ijebu Igbo as related to the underlying bedrocks. Unpublished B. Sc Geology Project, Olabisi Onabanjo University, Ago Iwoye.

Gibbs, R. J. (1970). Mechanisms controlling world chemistry. Science, 170, 1088-1090. http://dx.doi.org/10.1126/science.170.3962.1088

Hubbard, F. H. (1975). Precambrian crustal development in western Nigeria: indicators from the Iwo region. Geo. Soc. Am. Bull, 86, 548-554. http://dx.doi.org/10.1130/0016-7606(1975)86<548:PCDIWN>2.0.CO;2

Ipinmoroti, K. O. (1993). Determination of trace metals in fish associated water and soil sediments in fresh fish pond. Discovery and innovations, $5,138 \mathrm{p}$.

Jones, H. A., \& Hockey, R. D. (1964). The geology of part of southwestern Nigeria. Nigeria Min.Geol. and Metall. Soc., 1.

Mapanda, F., Mangwayana, E. N., \& Giller, K. E. (2005). The Effect of long term irrigation using waste water on heavy metal contents of soils under vegetables in Harare Zimbabwe. Agri. Ecosyst. Environ., 107, 151-165. http://dx.doi.org/10.1016/j.agee.2004.11.005

Odeyemi, I. B. (1993). A comparative study of remote sensing images of the structure of Okemesi fold belt Nigeria. I. T. C. J. $1931(1), 77-81$.

Oke, S. A. (2010). Hydrogeochemical assessment of influence of bedrock weathering on chemical character of shallow groundwater system: A case study of Abeokuta. Unpublished M. Sc Project, Department of Geology, University of Ibadan.

Okunlola, O. A., \& Okoroafor, E. R. (2009). Geochemical and petrogenetic features of Schistose rocks of the Okemesi fold belt, Southwestern, Nigeria. RMZ- materials and geoenvironment, 56(2), 148-162.

Olobaniyi, E. B., Ogala, J. E., \& Nfor, N. B. (2007). Hydrogeology and bacteriological investigation of groundwater in Agbor area, Southwestern Nigeria. Journal of Mining and Geology, 43(1), 79-89. http://dx.doi.org/10.4314/jmg.v43i1.18867

Olobaniyi, S. O. (2003). Geochemistry of semi pelitic schist of Isanlu area, southwestern Nigeria: Implication for the geodynamic evolution of the Egbe-Isanlu schist belt. Global Journal of Geological Science, 1(2), 113-127.

Oluduro, A. O., \& Adeyowe, B. I. (2007). Efficiency of moringa Oleifera seed extract on the microflora of surface and groundwater. Journal of plant Science, 6, 453-438.

Piper, A. M. (1944). A graphical procedure in geochemical interpretation of water analysis. Trans American Geophysical Union, 25, 914 - 923. http://dx.doi.org/10.1029/TR025i006p00914

Rahaman, M. A. (1976). Review of the basement geology of Southwestern Nigeria, in Geology of Nigeria. In C. A. Kogbe (Ed.) Geology of Nigeria (2 ${ }^{\text {nd }}$ Revised Ed., pp. 41-58). Elizabethan Pub. Co. Lagos.

Sadashivaiah, C., Ramakrishnaiah, C. R., \& Ranganna, G. (2008). Hyrochemical Analysis and Evaluation of Groundwater in Tumkur Taluk, Karnataka State, India. International Journal of Public Health, 5(3), 158-164. http://dx.doi.org/10.3390/ijerph5030158

Schoeller, H. I. (1962). Les eanx souterraines, meson and paris.

Tijani, M. N. (1994). Hydrogeochemical assessment of groundwater in Moro area, Kwara State. Nigeria Journal of Environmental Geology, 24, 194 - 202. http://dx.doi.org/10.1007/BF00766889 
Tijani, M. N. (2010). Groundwater system: A resource between the twin forces of nature and man. A faculty lecture delivered at the Faculty of Science, University of Ibadan. First Edition, Ibadan University Press.

Tijani, M. N., \& Nton, M. E. (2009). Hydraulic, textural and geochemical characteristics of the Ajali Formation, Anambra Basin, Nigeria: Implication for groundwater quality. Journal of Environmental Geology, 56, 935-951. http://dx.doi.org/10.1007/s00254-008-1196-1

Todd, D. K. (1980). Groundwater Hydrology (2nd Ed., 315p). New York: Wiley.

Tredoux, G., Tama, A. S., \& Engelbrecht, S. (2001). The increasing nitrate hazard in groundwater in the rural areas. Paper presented at Water Institute Conference. Sun City, South Africa.

WHO Guidelines for drinking water quality. World Health Organization publication (2004): Geneva, Switzerland. Wilcox, L. V. (1995). Classification and use of irrigation waters, US Department of Agriculture, Washington Dc.

\section{Copyrights}

Copyright for this article is retained by the author(s), with first publication rights granted to the journal.

This is an open-access article distributed under the terms and conditions of the Creative Commons Attribution license (http://creativecommons.org/licenses/by/3.0/). 